\title{
A cluster-randomized controlled trial to evaluate the effects of a simplified cardiovascular management program in Tibet, China and Haryana, India: study design and rationale
}

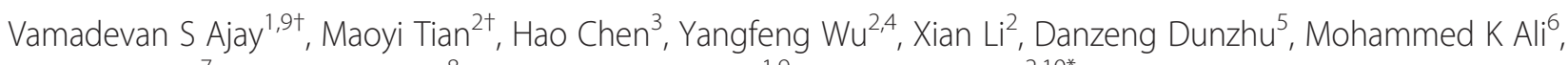
Nikhil Tandon ${ }^{7}$, Anand Krishnan ${ }^{8}$, Dorairaj Prabhakaran ${ }^{1,9}$ and Lijing L Yan $2,10^{*}$

\begin{abstract}
Background: In resource-poor areas of China and India, the cardiovascular disease burden is high, but availability of and access to quality healthcare is limited. Establishing a management scheme that utilizes the local infrastructure and builds healthcare capacity is essential for cardiovascular disease prevention and management. The study aims to develop, implement, and evaluate the feasibility and effectiveness of a simplified, evidence-based cardiovascular management program delivered by community healthcare workers in resource-constrained areas in Tibet, China and Haryana, India.

Methods/design: This yearlong cluster-randomized controlled trial will be conducted in 20 villages in Tibet and 20 villages in Haryana. Randomization of villages to usual care or intervention will be stratified by country. High cardiovascular disease risk individuals (aged 40 years or older, history of heart disease, stroke, diabetes, or measured systolic blood pressure of $160 \mathrm{mmHg}$ or higher) will be screened at baseline. Community health workers in the intervention villages will be trained to manage and follow up high-risk patients on a monthly basis following a simplified ' $2+2$ ' intervention model involving two lifestyle recommendations and the appropriate prescription of two medications. A customized electronic decision support system based on the intervention strategy will be developed to assist the community health workers with patient management. Baseline and follow-up surveys will be conducted in a standardized fashion in all villages. The primary outcome will be the net difference between-group in the proportion of high-risk patients taking antihypertensive medication pre- and post-intervention. Secondary outcomes will include the proportion of patients taking aspirin and changes in blood pressure. Process and economic evaluations will also be conducted.

Discussion: To our knowledge, this will be the first study to evaluate the effect of a simplified management program delivered by community health workers with the help of electronic decision support system on improving the health of high cardiovascular disease risk patients. If effective, this intervention strategy can serve as a model that can be implemented, where applicable, in rural China, India, and other resource-constrained areas.

(Continued on next page)
\end{abstract}

\footnotetext{
* Correspondence: lyan@georgeinstitute.org.cn

${ }^{\dagger}$ Equal contributors

${ }^{2}$ The George Institute for Global Health at Peking University Health Science

Center, Suite 1801, Tower B, Horizon Tower, No. 6 Zhichun Road, Haidian

District, Beijing 100088, China

${ }^{10}$ Duke Global Health Institute, and Global Heath Research Center, Duke Kunshan University, China No.1699 Zuchongzhi Road, Kunshan, Jiangsu

Province 215347, China

Full list of author information is available at the end of the article
}

C Biomed Central (c) 2014 Ajay et al.; licensee BioMed Central Ltd. This is an Open Access article distributed under the terms of the Creative Commons Attribution License (http://creativecommons.org/licenses/by/4.0), which permits unrestricted use, distribution, and reproduction in any medium, provided the original work is properly credited. The Creative Commons Public Domain Dedication waiver (http://creativecommons.org/publicdomain/zero/1.0/) applies to the data made available in this article, unless otherwise stated. 
(Continued from previous page)

Trial registration: The trial was registered in the clinicaltrials.gov database on 30 December, 2011 and the registration number is NCT01503814.

Keywords: Cardiovascular diseases prevention and control, Research design, Developing countries, Rural population, Community health workers, Risk reduction, Electronic decision support system

\section{Background}

An estimated 17 million people die of cardiovascular disease (CVD) each year with over $80 \%$ of these deaths occurring in low and middle-income countries [1]. Resource-poor areas in China and India, where CVD is already the leading cause of death, confront a rising disease burden with a strained healthcare system often inadequately equipped to deal with these problems [2-4]. Many of the risk factors strongly associated with CVD, including high blood pressure, tobacco use, and poor diet are among the top risk behaviors in these countries [5,6]. For example, in studies assessing hypertension in rural areas of Tibet, China, up to $14.9 \%$ of the population aged 15 years or older was found to be hypertensive, with some townships having rates as high as $55.9 \%$ in adults 40 years old and above $[7,8]$. The overall hypertension rate was found to be just as high in Haryana, India, with the urban slums of Faridabad District, Haryana similarly affected at $16.5 \%[9,10]$. Despite these high rates of hypertension, the number of patients receiving antihypertensive therapy in these areas remains low, and way below the averages seen in urban areas [7,11].

There are many well-established interventions such as lifestyle modification of known risk factors and effective, low-cost medications that can help avert the CVD burden in these economic and healthcare resource limited settings [12-21]. One particularly cost-effective approach is to identify and manage individuals at high cardiovascular disease risk in order to prevent or delay events [22-28]. This method has been applied and implemented in the Rural Andhra Pradesh Cardiovascular Prevention Study in India and the China Rural Health Initiative in northern China [29-31].

Community Health Workers (CHWs) are the members who received basic professional training to provide health and medical care for the community. Involvement of CHWs in disadvantaged populations has resulted in a significant improvement in accessing healthcare facilities, screening disease, monitoring community members' health status and adherence to treatment [32-35]. Task-shifting to CHWs has been proven as a costeffective and sustainable approach in chronic disease prevention and control, particularly in those areas with limited economic and healthcare resources [36]. More recently, with the aid of mobile phones, studies have shown a significant reduction in $\mathrm{CHW}$ training time, disease screening time and an enhanced user satisfaction [37]. A recent systematic review also shows that mobile phone based technology in low and middle income countries positively impacts the chronic disease management and its associated clinical outcomes [38].

Simplifying and extending the high-risk intervention strategy to suit areas with high CVD burden, but more limited economic and healthcare resources will be essential to addressing this problem in an effective and affordable way in these areas. China and India are two largest developing countries in the world that face similar challenges in terms of the burden of CVD and resource constraints in tackling these challenges. Yet, there are also many differences in the healthcare systems, socioeconomical environment and ethno-cultural customs that will make conducting a joint study illuminating and fruitful.

Therefore, we have designed a study to develop and evaluate the effects of implementing, in resource-scarce settings, a highly simplified but guideline-based program for cardiovascular management by $\mathrm{CHWs}$ with the aid of electronic decision support system in rural China and India (the SimCard study). This paper describes the research design and the rationale of the study.

\section{Methods/design}

The SimCard study is a cluster-randomized controlled interventional trial to be conducted in rural areas of Tibet, China and Haryana, India. The design, implementation and reporting of the study follow recommendations from CONSORT statement on cluster-randomized trials [39]. The trial was registered in the clinicaltrials. gov database on 30 December, 2011 and the registration number is NCT01503814. The study protocol received ethics approval from the institutional review boards at Peking University Health Sciences Center, China, the Public Health Foundation of India, India, and Duke University, USA. Informed consent will be obtained from each study participant.

\section{Study sites}

Two counties in the Tibet autonomous region located in Southwest China - Gongbujiangda and Linzhou and one tehsil - Ballabgarh in Faridabad District from Haryana State in northwestern India will participate in 
the study. Villages from these locations will be selected to take part in this study based on the site selection criteria of (1) high CVD burden, (2) limited healthcare resources, (3) having available existing CHWs or qualified candidates who can be trained to fulfill that role, and (4) local government support. A total of 40 villages will be recruited across the two countries, 20 in each country.

\section{Randomization}

In each country, villages will be randomized to receive the intervention or usual care. Randomization will be stratified by country. In China, all selected villages will be randomized by a 1:1 ratio to the intervention and control group with stratification by county and township (the administrative unit managing the villages). In India, randomization will not be stratified by any geographical locations. Randomization in both countries will be conducted by a study staff not involved in the intervention through a central computerized process. Group allocation will be concealed until after the baseline data collection is completed.

\section{Study population}

Participants at CVD high risk will be eligible for study enrollment. In this study, 'high-risk' is defined as older age ( 40 years old and above) with a history of any of the following conditions: coronary heart disease, stroke (ischemic, hemorrhagic, or unknown/unspecified type), diabetes mellitus, and/or having a systolic blood pressure $\geq 160 \mathrm{mmHg}$ at two different time points measured in the same day during the baseline survey.

Exclusion criteria include having CVD-related complications that cannot be managed in a primary care setting, having a malignancy or life-threatening disease; individuals who are bed-ridden, currently participating in any other clinical trials, unable to stay in the village longer than 8 months in a year, and planning to move in the next year.

\section{Screening}

Before the start of the baseline data collection, a survey will be conducted in all participating villages to screen for high-risk individuals. All village residents 40 years and above will be invited to participate in the survey, which will include a brief questionnaire on disease history and measuring blood pressure twice. All high-risk individuals screened out through this process according to the definition described above, a target minimum of 50 in each village, will be invited to participate in the study.

\section{Intervention and control Intervention}

The intervention scheme adapts and simplifies international and applicable national clinical guidelines for hypertension and cardiovascular disease management for use by local CHWs to manage cardiovascular high-risk patients in an easily implementable, low cost, and medically effective way $[23,40]$. Local CHWs will be trained to provide basic monthly follow-up care to identified highrisk patients, and refer them to higher level healthcare facilities when necessary. The intervention strategy consists of the following:

\section{' $2+2$ ' intervention model}

Taking into consideration the local health behaviors and resources, a simplified management model is devised that focuses on two therapeutic lifestyle recommendations (smoking cessation and salt reduction) and the appropriate prescription of two types of accessible, highly effective, generally safe, and low-cost drugs (blood pressure lowering drugs and aspirin) [41-46]. In India, as stipulated by the ethic committee, the drugs used in the intervention arm will be also made available to the primary care facilities serving the control villages. Coined the " $2+2$ " intervention model, the scheme targets two prevalent risk behaviors with established relationships to CVD while providing instruction on the prescription of two types of basic drugs that have well-documented effects on CVD risk reduction. All individuals in the intervention group will receive therapeutic lifestyle recommendations when applicable, but depending on each individual's medical situation may be prescribed both, one, or none of the two types of drugs. These interventions will be delivered by CHWs and they will provide monthly follow-up to all high-risk patients under their care.

\section{Systematic training of local CHWs}

Enhancing the capacity of local CHWs through systematic training is a key feature of the intervention model. Training of local CHWs in the intervention group will consist of an initial training on the intervention protocol, including education on the targeted CVD lifestyle risk factors and the medications being utilized, followed by refresher training about 1-3 months after the intervention begins. Only CHWs who pass the initial evaluation examination can take part in implementing the intervention. After the training, CHWs will be provided with a list of high-risk individuals screened out at baseline for them to contact and manage according to the protocol.

In Tibet, China, "village doctors" who are not physicians but have basic professional training and can prescribe medications will be chosen to be the CHWs to implement this management plan. In rural India, local women will be recruited and trained to be the study's $\mathrm{CHWs}$. Because the Indian CHWs have no prior medical experience, they will be partnered with licensed physicians from nearby primary health care centers who are 
responsible for the prescription duties based on the CHW' regular assessments of their assigned high-risk patients.

\section{Electronic decision support}

An electronic decision support (EDS) component will be incorporated in this study to assist the CHWs on the follow up and management of their high-risk patients during the one-year intervention period. The EDS system is a smartphone or tablet-based android application that will be developed and pilot tested based on the ' $2+2$ ' intervention model and consists of prompts regarding the patient's medical history, new conditions, medication usage, current lifestyle habits, blood pressure, and the appropriateness for prescribing any of the target medications. The follow-up records entered into the device can be easily uploaded by CHWs to a central server to generate performance indicators and provide feedback to help improve the quality of care. In India, the EDS system has a desktop component for the use of physicians to prescribe drugs in the intervention arm.

\section{Performance feedback}

Tiered monetary payments based on key performance indicators will be given to CHWs on a recurring basis. Examples of key performance indicators include the number and percentage of high-risk individuals receiving regular follow-up, lifestyle advices, and medications.

\section{Control}

Villages in the control group will continue their usual practices without any of the above mentioned interventions. Usual practices will include any other government or project-initiated lifestyle, hypertension or cardiovascular management programs.

\section{Outcome evaluation}

\section{Baseline and post-intervention survey}

The outcome evaluation for this study will be derived from data collected from the baseline survey and postintervention follow-up survey of all eligible and consenting high-risk patients in the intervention and control villages. Both survey questionnaires will be identical, and include information on patient's demographics, disease history, lifestyle behaviors, medication use, primary healthcare services utilization, hospitalizations, and medical care related expenses in the past year. Height, weight, waist circumference, and blood pressure will be measured at that time of the survey. Surveys in both countries will be conducted by trained personnel according to the same standardized operating procedures. The questionnaires used in China and India will be kept nearly identical except for a few variations to fit the cultural context and target the specific lifestyles of the two peoples in these countries such as different types of tobacco use (See Table 1 for survey indicators).

The primary outcome will be the net difference between the intervention and control groups in the pre- and postintervention change in the proportion of high-risk individuals treated with anti-hypertensive medication, obtained through the two surveys described above. This process indicator is chosen for its close association with the intervention scheme, effect on lowering high blood pressure, and its excellent power.

A number of secondary outcomes will also be evaluated, including the net difference between the intervention and control groups in the pre- and post-intervention change in the proportion of high-risk individuals treated with aspirin, the net difference in mean blood pressure from baseline to follow-up in high-risk individuals between the intervention and control villages, as well as hypertension awareness, treatment, and control rates. Awareness of modifiable lifestyle risk behaviors will also be assessed, specifically on the proportion of high-risk individuals aware of the harms of smoking and consumption of a high salt diet. Finally, the proportion of high-risk individuals receiving five or more follow-up visits from CHWs will also be calculated.

\section{Sample size}

Key underlying assumptions for this study are that there will be 20 intervention villages and 20 control villages with 50 consenting high-risk patients in each village and an estimated total of 2,000 high-risk individuals, an intra-cluster correlation coefficient (ICC) of 0.01 or $0.02[36,47]$, and two-sided alpha of 0.05 . For the primary outcome, assuming the proportion of antihypertensive medication prescriptions in the control

\begin{tabular}{lll}
$\begin{array}{l}\text { Table } 1 \text { Indicators followed in baseline and follow-up } \\
\text { survey }\end{array}$ & Measures & Methods \\
\hline Indicators & Tobacco & \\
\hline & Alcohol* & Questionnaire \\
Behavioral & Physical activity & \\
& Salt consumption & \\
& Height & Stadiometer \\
& Weight & Weighing machine \\
Anthropometric & Waist circumference & Measuring tape \\
& $\begin{array}{l}\text { Blood pressure } \\
\text { Compliance }\end{array}$ & Electronic BP monitor \\
& $\begin{array}{l}\text { Awareness, Risk } \\
\text { factor control }\end{array}$ & Questionnaire \\
Treatment history, Health & Service utilization & \\
services, Health care costs & Treatment cost & \\
&
\end{tabular}

*: Not covered in Tibet, China site. 
villages is $20 \%$ (conservative as preliminary data shows it to be $<10 \%$ in the target study sites), the power to detect a $10 \%$ difference in the primary outcome of anti-hypertensive medication use is excellent (>90\%) with an ICC of 0.01, and similarly high with an ICC of 0.02 . Assuming a standard deviation of change in systolic blood pressure of $15 \mathrm{mmHg}$ among the high-risk individuals, the power to detect a $3 \mathrm{mmHg}$ net difference in this secondary outcome between the intervention and control group pre-post differences will be $>90 \%$ with an ICC of 0.01 ( $>90 \%$ as well if ICC $=0.02$ ).

\section{Statistical analysis}

All analyses will be conducted at individual level according to intention-to-treat principles, and take into account the stratification design and cluster effects. For the primary outcome, anti-hypertensive medication use, log-binomial models with random effects for villages to account for clustering and random effects for intercept to account for the pre-post correlations within individuals and a fixed effect for country to account for the stratified study design will be used. Other than the main effect from intervention and time, an interaction term between intervention and time will be added in the model to test if the slope of the pre-post changes differs by intervention or control group. To account for the heterogeneity between two countries, the two-way interaction of the country variable with intervention and time will be included into the above models. In the case of significant interaction existing, the separate analysis by country will be conducted afterward. The net effect from intervention will be reported as difference-in-difference of proportions together with model-based 95\% confidence intervals obtained by transforming back from models. For continuous outcomes, such as systolic blood pressure, similar strategies will be adopted but linear models instead of log-binomial models will be used. Some adjusted analysis will also be performed to account for potential unbalanced baseline information. Other pre-specified subgroup will include analyses by sex and age (40-59 vs. 60 years or older) using similar strategy for the country variable.

\section{Process and economic evaluation}

Process evaluation will be conducted at the end of the intervention program to investigate the extent to which the intervention has been implemented as designed, and to identify the facilitators and barriers to the implementation of the intervention program at each level of the study. We will conduct face-to-face, semi-structured interviews by independent interviewers. Interviewees include the CHWs, high-risk individuals in the selected villages, local project administrators, and government officials. A standardized interview guideline will be developed that contains a mixture of close- and open-ended questions. All interviews will be audio-recorded, transcribed and analyzed using a qualitative descriptive interpretive approach combing thematic content analysis and constant comparison methods facilitated by QSR NVIVO 10.0 data management software. The economic evaluation will help determine the economic feasibility of expanding such an intervention using data collected on healthcare services usage and medication use as obtained from the follow-up surveys.

\section{Discussion}

CVD is a global problem that affects developed and developing countries alike. Areas with constrained economic or healthcare resources face an even tougher challenge as the awareness of CVD is lacking and trained healthcare professionals are limited. The SimCard study in China and India aims to evaluate a simplified, multifaceted, and innovative intervention scheme for CVD prevention and control in resource-scarce settings. To the best of our knowledge, SimCard is the first study to incorporate an EDS system delivered by the CHWs for CVD management in these two countries. The findings of the study will provide evidence on the feasibility, acceptability and effectiveness of a simplified yet guideline-based intervention program delivered by the CHWs in resource constrained areas.

The study design aims to maximize the feasibility of the intervention through a simplified culturally-tailored CVD prevention and management plan based on the guidelines. Despite there being well-established national guidelines and policies on effective strategies to prevent and control CVD in China and India, its uptake into routine medical care remains very limited in resource constrained areas. This is primarily due to the complexity of the guideline, the lack of awareness of the guidelines by the CHWs and CHW's limited capacity to implement the guidelines. CHWs who will be utilizing the simplified management scheme descried in this study will be thoroughly trained and tested for competency on the indications, contraindications and side effects of the medications. CHWs will also be trained to provide therapeutic lifestyle recommendations as applicable. CHWs will be provided incentives based on the key performance indicators such as the regular follow-up rate, the percentage of high-risk individuals receiving lifestyle advices and other measurements.

The intervention is also designed to be innovative. By 2013, there were over 2 billion mobile phone subscribers in China and India [48]. This accounts for a staggering one third of the world's mobile users [48]. Smartphones and tablets with their advanced multimedia capability and connectivity to the internet are rapidly expanding in the developing countries' market. These mobile devices 
are rapidly becoming the dominant mode of accessing the internet and for example, China represents $22 \%$ of the global smartphone subscribers [49]. China has surpassed the U.S.A. to become the world's top country for active Android and iOS users. Given the near ubiquity of mobile phones and the exponential growth of smart phones and tablets in Chinese and Indian markets, these devices represent one of the few hardware products available with the potential to transform the delivery of health care. There is growing evidence showing smartphone or tablet based EDS system can improve patient care in both developed and developing countries [38,50]. Therefore, we incorporate the smartphone or tablet based EDS system into the intervention aiming to help the CHWs better follow-up and manage their high CVD risk patients.

Such a study is needed in both rural China and India where the prevalence of hypertension and CVD has been increasing rapidly and has reached epidemic proportions. The healthcare systems in both countries by and large are still acute care-oriented and ill-equipped to tackle these challenges. Shifting to and sharing of certain preventive tasks with CHWs is one important strategy that both countries need to evaluate for effectiveness and future scale-up if possible. In addition, implementation of the intervention needs to adapt to local contexts in order to be successful. China and India (in our study, Tibet and Haryana, respectively) possess distinctive features in primary healthcare delivery models, local cultural values and practices, and even regulations and governance of research studies. We anticipate this joint study in two countries will shed light on how this adaptation process can take place and how these differences may influence the results of the study. For example, in Tibet, Tibetan medicine is a widely believed system that can sometimes be at odds with Western medicine. In north India, women do not generally interact with non-family men. This gender relations issue will potentially pose some constraints to the Indian CHWs, who will all be female, in carrying out the management plan among male high-risk patients, which can be addressed with involvement of women from the patient's household. Many strategies and standardized operation procedures will be in place to ensure the quality of the implementation of the study protocol while taking into consideration differences between the two countries.

The burden of non-communicable chronic diseases is rapidly rising, but many areas lack the economic and healthcare resources to effectively deal with these widespread health problems. While evidence-based national and international guidelines on managing chronic conditions such as hypertension, coronary heart disease, and stroke are well-established, cost-effective approaches suitable for adoption in resource-constrained settings have not been adequately investigated. The SimCard study aims to address the highly prevalent problem of CVD in these remote and resource- limited areas that have not received much attention so far. The results of the study are expected both to advance scientific knowledge and to provide translational evidence necessary for sound policy making to address the CVD problem in these settings, in China, India, and where applicable, to other countries.

\section{Abbreviations}

CVD: Cardiovascular disease; CHW: Community health worker; EDS: Electronic decision support.

\section{Competing interests}

The authors declare that they have no competing interests.

\section{Authors' contributions}

MT participated in study design and drafted the manuscript. HC, YW, XL, DD, MKA, NT, AK and DP participated in study design and critical review of the manuscript. VSA and LLY conceived of the study, participated in its design and critical review of the manuscript. All authors read and approved the final manuscript.

\section{Acknowledgements}

We would like to thank Drs. Eric Peterson, Elizabeth DeLong, and Anushka Patel for their valuable inputs. We would also like to thank KaWing Cho and Ruilai Li for their contribution on drafting the manuscript.

\section{Funding}

This project has been funded in part with Federal funds from the United States National Heart, Lung, and Blood Institute, National Institutes of Health, Department of Health and Human Services, under Contract No. HHSN268200900027C. Additional support was received from the UnitedHealth Group Chronic Disease Initiative. LL Yan is also supported by the National Natural Sciences Foundation of China grants (71110107025, 71233001), NIH R01 grant (R01AG023627) and UNFPA.

\section{Author details}

${ }^{1}$ CoE-CARRS, Public Health Foundation of India, 4th Floor, Plot No - 44, Sector 44, Gurgaon, Haryana 122002, India. ${ }^{2}$ The George Institute for Global Health at Peking University Health Science Center, Suite 1801, Tower B, Horizon Tower, No. 6 Zhichun Road, Haidian District, Beijing 100088, China. ${ }^{3}$ Department of Cardiology, Beijing Hospital, No. 1 Dahua Road, Dongcheng District, Beijing 100730, China. ${ }^{4}$ Department of Epidemiology and

Biostatistics, Peking University School of Public Health, No. 38 Xueyuan Road, Haidian District, Beijing 100083, China. ${ }^{5}$ Tibet University, Jiangsu Road, Chengguan District, Lhasa 850000, China. ${ }^{6}$ Rollins School of Public Health, Emory University, 1518 Clifton Road, Atlanta, GA 30322, USA. ${ }^{7}$ Department of Endocrinology and Metabolism, All India Institute of Medical Sciences, Ansari Nagar, New Delhi 110029, India. ${ }^{8}$ Department of Community Medicine, All India Institute of Medical Sciences, Ansari Nagar, New Delhi 110029, India. ${ }^{9}$ Centre for Chronic Disease Control, 4th Floor, Plot \# 47, Sector-44, Gurgaon, New Delhi 122002, India. ${ }^{10}$ Duke Global Health Institute, and Global Heath Research Center, Duke Kunshan University, China No.1699 Zuchongzhi Road, Kunshan, Jiangsu Province 215347, China.

Received: 28 August 2014 Accepted: 2 September 2014 Published: 6 September 2014

\section{References}

1. World Health Organization PHAoC: Preventing chronic diseases: a vital investment. Geneva; Ottawa: World Health Organization; Public Health Agency of Canada; 2005.

2. Joshi R, Jan S, Wu Y, MacMahon S: Global inequalities in access to cardiovascular health care: our greatest challenge. J Am Coll Cardiol 2008, 52(23):1817-1825.

3. Reddy KS: Cardiovascular disease in non-Western countries. N Eng/ J Med 2004, 350(24):2438-2440. 
4. Yusuf S, Reddy S, Ounpuu S, Anand S: Global burden of cardiovascular diseases: part I: general considerations, the epidemiologic transition, risk factors, and impact of urbanization. Circulation 2001, 104(22):2746-2753.

5. Liu L: Cardiovascular diseases in China. Biochem Cell Bio/ 2007, 85(2):157-163.

6. Mackay J, Mensah GA, Mendis S, Greenlund K: The Atlas of Heart Disease and Stroke. World Health Organization: Geneva; 2004

7. Gusanglamu P, Cai YX, Rendan L: Hypertension prevalence, awareness, treatment and control rates in agricultural and pastoral areas in Tibet, China. Zhong Guo Yi Xue Zhi Nan 2010, 8(027):79-81.

8. Zhao X, Li S, Ba S, He F, Li N, Ke L, Li X, Lam C, Yan LL, Zhou Y, Wu Y: Prevalence, awareness, treatment, and control of hypertension among herdsmen living at 4,300 m in Tibet. Am J Hypertens 2012, 25(5):583-589.

9. Anand K, Shah B, Gupta V, Khaparde K, Pau E, Menon GR, Kapoor SK: Risk factors for non-communicable disease in urban Haryana: a study using the STEPS approach. Indian Heart J 2008, 60(1):9-18.

10. Anand K, Shah B, Yadav K, Singh R, Mathur P, Paul E, Kapoor SK: Are the urban poor vulnerable to non-communicable diseases? A survey of risk factors for non-communicable diseases in urban slums of Faridabad. Nat Med J India 2007, 20(3):115-120.

11. Wu Y, Huxley R, Li L, Anna V, Xie G, Yao C, Woodward M, Li X, Chalmers J, Gao R, Kong L, Yang X: Prevalence, awareness, treatment, and control of hypertension in China: data from the China National Nutrition and Health Survey 2002. Circulation 2008, 118(25):2679-2686.

12. The Intercollegiate Working Party for Stroke: National Clinical Guidelines for Stroke. Update. London: Royal College of Physicians; 2002.

13. Kernan WN, Ovbiagele B, Black HR, Bravata DM, Chimowitz MI, Ezekowitz MD, Fang MC, Fisher M, Furie KL, Heck DV, Johnston SC, Kasner SE, Kittner SJ, Mitchell PH, Rich MW, Richardson D, Schwamm LH, Wilson JA, American Heart Association Stroke Council CoC, Stroke Nursing CoCC, Council on Peripheral Vascular D: Guidelines for the prevention of stroke in patients with stroke and transient ischemic attack: a guideline for healthcare professionals from the American Heart Association/American Stroke Association. Stroke 2014, 45(7):2160-2236

14. China guideline of hypertension updated 2005 Progression in the diagnosis and management of hypertension: China Hypertension League. 2005

15. Beilin LJ: Non-pharmacological management of hypertension: optimal strategies for reducing cardiovascular risk. J Hypertens Supp/ 1994, 12(10):S71-S81.

16. Clark AM, Hartling L, Vandermeer B, McAlister FA: Meta-analysis: secondary prevention programs for patients with coronary artery disease. Ann Intern Med 2005, 143(9):659-672.

17. Cutler JA, Follmann D, Allender PS: Randomized trials of sodium reduction: an overview. Am J Clin Nutr 1997, 65(2 Suppl):643S-651S

18. Cutler JA, Follmann D, Elliott P, Suh I: An overview of randomized trials of sodium reduction and blood pressure. Hypertension 1991 17 (1 Suppl):127-133.

19. European Society of Hypertension-European Society of Cardiology Guidelines C: 2003 European Society of Hypertension-European Society of Cardiology guidelines for the management of arterial hypertension. J Hypertens 2003, 21(6):1011-1053

20. Organization WH: Global strategy on diet, physical activity and health, F.-S.W.H. Assembly, Editor. [http://www.who.int/dietphysicalactivity/strategy/eb11344/ strategy_english_web.pdf]

21. Williams B, Poulter NR, Brown MJ, Davis M, Mclnnes GT, Potter JF, Potter JF, Sever PS, Mc GTS, British Hypertension S: Guidelines for management of hypertension: report of the fourth working party of the British Hypertension Society, 2004-BHS IV. J Hum Hypertens 2004, 18(3):139-185.

22. National Vascular Disease Prevention Alliance: Guidelines for the Assessment of Absolute Cardiovascualr Disease Risk. Melbourne: National Heart Foundation of Australia; 2009

23. Chobanian AV, Bakris GL, Black HR, Cushman WC, Green LA, Izzo JL Jr, Jones DW, Materson BJ, Oparil S, Wright JT Jr, Roccella EJ, National Heart L, Blood Institute Joint National Committee on Prevention DE, Treatment of High Blood P, National High Blood Pressure Education Program Coordinating C: The seventh report of the joint national committee on prevention, detection, evaluation, and treatment of high blood pressure: the JNC 7 report. JAMA 2003, 289(19):2560-2572. doi:10.1001/jama.289.19.2560. PubMed PMID: 12748199

24. Gaziano TA, Opie LH, Weinstein MC: Cardiovascular disease prevention with a multidrug regimen in the developing world: a cost-effectiveness analysis. Lancet 2006, 368(9536):679-686.
25. Gaziano TA, Steyn K, Cohen DJ, Weinstein MC, Opie LH: Cost-effectiveness analysis of hypertension guidelines in South Africa: absolute risk versus blood pressure level. Circulation 2005, 112(23):3569-3576.

26. Murray CJ, Lauer JA, Hutubessy RC, Niessen L, Tomijima N, Rodgers A, Lawes CM, Evans DB: Effectiveness and costs of interventions to lower systolic blood pressure and cholesterol: a global and regional analysis on reduction of cardiovascular-disease risk. Lancet 2003, 361(9359):717-725.

27. Reddy KS: Cardiovascular diseases in the developing countries: dimensions, determinants, dynamics and directions for public health action. Public Health Nutr 2002, 5(1A):231-237.

28. Wu Y, Liu X, Li X, Li Y, Zhao L, Chen Z, Li Y, Rao X, Zhou B, Detrano R, Liu K, Cardiovascular U-PCSo, Cardiopulmonary Epidemiology Research G, China Multicenter Collaborative Study of Cardiovascular Epidemiology Research G: Estimation of 10-year risk of fatal and nonfatal ischemic cardiovascular diseases in Chinese adults. Circulation 2006, 114(21):2217-2225.

29. Chow CK, Joshi R, Gottumukkala AK, Raju K, Raju R, Reddy S, Macmahon S, Neal B: Rationale and design of the Rural Andhra Pradesh Cardiovascular Prevention Study (RAPCAPS): a factorial, cluster-randomized trial of 2 practical cardiovascular disease prevention strategies developed for rural Andhra Pradesh. India Am Heart J 2009, 158(3):349-355.

30. Joshi R, Chow CK, Raju PK, Raju KR, Gottumukkala AK, Reddy KS, Macmahon S, Heritier S, Li Q, Dandona R, Neal B: The Rural Andhra Pradesh Cardiovascular Prevention Study (RAPCAPS): a cluster randomized trial. J Am Coll Cardiol 2012, 59(13):1188-1196.

31. Yan LL, Fang W, Delong E, Neal B, Peterson ED, Huang Y, Sun N, Yao C, Li X, MacMahon S, Wu Y: Population impact of a high cardiovascular risk management program delivered by village doctors in rural China: design and rationale of a large, cluster-randomized controlled trial. BMC Public Health 2014, 14:345.

32. Allen JK, Dennison-Himmelfarb CR, Szanton SL, Bone L, Hill MN, Levine DM, West M, Barlow A, Lewis-Boyer L, Donnelly-Strozzo M, Curtis C, Anderson K: Community Outreach and Cardiovascular Health (COACH) Trial: a randomized, controlled trial of nurse practitioner/community health worker cardiovascular disease risk reduction in urban community health centers. Circ Cardiovasc Qual Outcomes 2011, 4(6):595-602

33. Chatterjee S, Naik S, John S, Dabholkar H, Balaji M, Koschorke M, Varghese M, Thara R, Weiss HA, Williams P, McCrone P, Patel V, Thornicroft G: Effectiveness of a community-based intervention for people with schizophrenia and their caregivers in India (COPSI): a randomised controlled trial. Lancet 2014, 383(9926):1385-1394.

34. Dye CJ, Williams JE, Evatt JH: Improving Hypertension Self-Management With Community Health Coaches, Health promotion practice. 2014.

35. Ursua RA, Aguilar DE, Wyatt LC, Katigbak C, Islam NS, Tandon SD, Nur PR, Van Devanter N, Rey MJ, Trinh-Shevrin C: A community health worker intervention to improve management of hypertension among Filipino Americans in New York and New Jersey: a pilot study. Ethn Dis 2014 24(1):67-76.

36. Jafar TH, Hatcher J, Poulter N, Islam M, Hashmi S, Qadri Z, Bux R, Khan A, Jafary FH, Hameed A, Khan A, Badruddin SH, Chaturvedi N, Hypertension Research G: Community-based interventions to promote blood pressure control in a developing country: a cluster randomized trial. Ann Intern Med 2009, 151(9):593-601.

37. Surka S, Edirippulige S, Steyn K, Gaziano T, Puoane T, Levitt N: Evaluating the use of mobile phone technology to enhance cardiovascular disease screening by community health workers. Int J Med Inform 2014, 83(9):648654.

38. Beratarrechea A, Lee AG, Willner JM, Jahangir E, Ciapponi A, Rubinstein A: The impact of mobile health interventions on chronic disease outcomes in developing countries: a systematic review. Telemed J E-health: Offic J Am Telemed Assoc 2014, 20(1):75-82.

39. Meade TW, Wald N, Collins R: CONSORT statement on the reporting standards in clinical trials. Recommendations are inappropriate for trial reports. BMJ 1997, 314(7087):1126.

40. Liu L: Chinese national hypertension prevention guideline 2010. Lisheng Liu 2011, 19(8):701-708.

41. Anderson TJ, Gregoire J, Hegele RA, Couture P, Mancini GB, McPherson R, Francis GA, Poirier P, Lau DC, Grover S, Genest J Jr, Carpentier AC, Dufour R, Gupta M, Ward R, Leiter LA, Lonn E, Ng DS, Pearson GJ, Yates GM, Stone JA, Ur E: 2012 update of the Canadian Cardiovascular Society guidelines for the diagnosis and treatment of dyslipidemia for the prevention of cardiovascular disease in the adult. Can J Cardio/ 2013, 29(2):151-167. 
42. Goff DC Jr, Lloyd-Jones DM, Bennett G, Coady S, D'Agostino RB, Gibbons R, Greenland P, Lackland DT, Levy D, O'Donnell CJ, Robinson JG, Schwartz JS, Shero ST, Smith SC Jr, Sorlie P, Stone NJ, Wilson PW, Jordan HS, Nevo L, Wnek J, Anderson JL, Halperin JL, Albert NM, Bozkurt B, Brindis RG, Curtis LH, DeMets D, Hochman JS, Kovacs RJ, Ohman EM, et al: 2013 ACC/AHA guideline on the assessment of cardiovascular risk: a report of the American College of Cardiology/American Heart Association Task Force on Practice Guidelines. Circulation 2014, 129(25 Suppl 2):S49-S73.

43. Gupta R, Deedwania P: Interventions for cardiovascular disease prevention. Cardiol Clin 2011, 29(1):15-34.

44. Perk J, De Backer G, Gohlke H, Graham I, Reiner Z, Verschuren WM, Albus C, Benlian P, Boysen G, Cifkova R, Deaton C, Ebrahim S, Fisher M, Germano G, Hobbs R, Hoes A, Karadeniz S, Mezzani A, Prescott E, Ryden L, Scherer M, Syvanne M, Op Reimer WJ, Vrints C, Wood D, Zamorano JL, Zannad F, European Association for Cardiovascular P, Rehabilitation: European guidelines on cardiovascular disease prevention in clinical practice (version 2012): the fifth joint task force of the European society of cardiology and other societies on cardiovascular disease prevention in clinical practice (constituted by representatives of nine societies and by invited experts). Int I Behav Med 2012, 19(4):403-488.

45. Shroufi A, Chowdhury R, Anchala R, Stevens S, Blanco P, Han T, Niessen L, Franco OH: Cost effective interventions for the prevention of cardiovascular disease in low and middle income countries: a systematic review. BMC Public Health 2013, 13:285.

46. Tang L, Patao C, Chuang J, Wong ND: Cardiovascular risk factor control and adherence to recommended lifestyle and medical therapies in persons with coronary heart disease (from the National Health and Nutrition Examination Survey 2007-2010). Am J Cardiol 2013, 112(8):1126-1132.

47. Parker DR, Evangelou E, Eaton CB: Intraclass correlation coefficients for cluster randomized trials in primary care: the cholesterol education and research trial (CEART). Contemp Clin Trials 2005, 26(2):260-267.

48. Reuters. In 2013. http://www.reuters.com/article/2013/12/20/chinamobilesubscribers-idUSL4N0J51ZN20131220.

49. Styles K: http://mobilemarketingmagazine.com/382m-smartphone-users-china/

50. Kawamoto K, Houlihan CA, Balas EA, Lobach DF: Improving clinical practice using clinical decision support systems: a systematic review of trials to identify features critical to success. BMJ 2005, 330(7494):765.

doi:10.1186/1471-2458-14-924

Cite this article as: Ajay et al: A cluster-randomized controlled trial to evaluate the effects of a simplified cardiovascular management program in Tibet, China and Haryana, India: study design and rationale. BMC Public Health 2014 14:924.

\section{Submit your next manuscript to BioMed Central and take full advantage of:}

- Convenient online submission

- Thorough peer review

- No space constraints or color figure charges

- Immediate publication on acceptance

- Inclusion in PubMed, CAS, Scopus and Google Scholar

- Research which is freely available for redistribution 